\title{
Optical Rotatory Dispersion Measurement of D-Glucose with Fixed Polarizer Analyzer Accessory in Conventional Spectrophotometer
}

\author{
Alfons Penzkofer \\ Faculty of Physics, University of Regensburg, Regensburg, Germany. \\ Email: alfons.penzkofer@ur.de \\ Received October $24^{\text {th }}, 2013$; revised November $24^{\text {th }}, 2013$; accepted December $7^{\text {th }}, 2013$ \\ Copyright (C) 2013 Alfons Penzkofer. This is an open access article distributed under the Creative Commons Attribution License, \\ which permits unrestricted use, distribution, and reproduction in any medium, provided the original work is properly cited.
}

\begin{abstract}
In the sample compartment of a conventional spectrophotometer, mounting of a polarizer before sample and an analyzer behind sample allows the determination of the optical rotatory dispersion of optical active media by measurement of the transmission ratio of crossed and parallel arranged polarizer and analyzer. A formula for the determination of the angle of rotation is derived from the transmission ratio. The arrangement is applied to determine the molar optical rotation of D-glucose in water in the wavelength range from $220 \mathrm{~nm}$ to $820 \mathrm{~nm}$.
\end{abstract}

Keywords: Optical Activity; Optical Rotatory Dispersion; Specific Optical Rotation; Molar Optical Rotation; Polarimeter; Spectropolarimeter; Grape Sugar; D-Glucose

\section{Introduction}

Substances with handed (chiral) structure are optically active (circular birefringent) [1-5]. They have different refractive index dispersion, $n(\lambda)$, for right and left circular polarized light, i.e. $n_{r}(\lambda) \neq n_{1}(\lambda)$ (circular birefringence), and light absorption is different for right and left circular polarized light, i.e. $\sigma_{r}(\lambda) \neq \sigma_{1}(\lambda)$ or $\varepsilon_{r}(\lambda) \neq \varepsilon_{1}(\lambda)$ (circular dichroism, $\sigma$ is absorption cross-section, $\varepsilon=N_{A} \sigma /[1000 \ln (10)]$ is molar decadic absorptivity, $\lambda$ is wavelength). Linear polarized light can be thought to be composed of equal amount of right and left circular polarized light. Optical active substances cause rotation of the polarization plane of linear polarized light in their transparency region due to different speed of right and left circular polarized light. In the absorption region linear polarized light becomes additionally elliptically polarized because of different attenuation of right and left circular polarized light.

The rotation of polarization at a fixed wavelength is generally measured with a polarimeter consisting of a monochromatic light source, an entrance polarizer, space for the sample, an exit polarizer (analyzer) on a rotation stage, and a detector [6]. The analyzer is rotated to crossed position (blocking of linear polarized light trans- mission) in accordance of the rotation of the linear polarization of the optical active sample under investigation. The optical rotatory dispersion (wavelength dependence of optical rotation) is measured with spectropolarimeters consisting of a broadband light source, a spectrometer, and a polarimeter [6].

Here the polarizer and analyzer are oriented perpendicular $(\perp)$ and then oriented parallel $(\|)$. In both cases the transmission through polarizer-sample - analyzer $\left(T_{\perp}\right.$ and $\left.T_{\|}\right)$is measured, and the angle of polarization rotation $\phi$ is calculated from the transmission ratio $T_{\perp} / T_{\|}$. A formula for the relation between $\phi$ and $T_{\perp} / T_{\|}$ is derived. The polarizer-sample - analyzer arrangement may be illuminated with a monochromatic light source (laser, light emitting diode, lamp with interference filter) for determination of the optical polarization rotation at a fixed wavelength (polarimeter function), or it may be assembled into the sample chamber of a conventional spectrophotometer to determine the wavelength dependence of the optical polarization rotation (spectropolarimeter function).

\section{Experimental}

The experimental arrangement for the determination of 
the optical rotation of polarization at a fixed wavelength is shown in the top part of Figure 1(a). The light source (here a He-Ne laser with $\lambda=632.8 \mathrm{~nm}$ ) emits monochromatic light along the z-direction through the polarizer (P1) - sample (S) - analyzer (P2) arrangement. The polarizer orientations and the optical polarization rotations of right (dextro) rotatory and left (laevo) rotatory samples are illustrated in the bottom part of Figure 1(a).

The light transmission through the P1-S-P2 arrangement is given by

$$
T=\frac{S_{\text {out }}}{S_{\text {in }}}=T_{\mathrm{P} 1} T_{\mathrm{S}} T_{\mathrm{P} 2},
$$

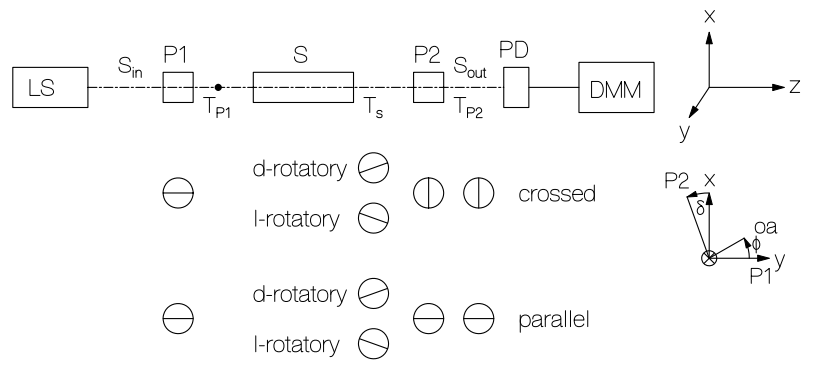

(a)

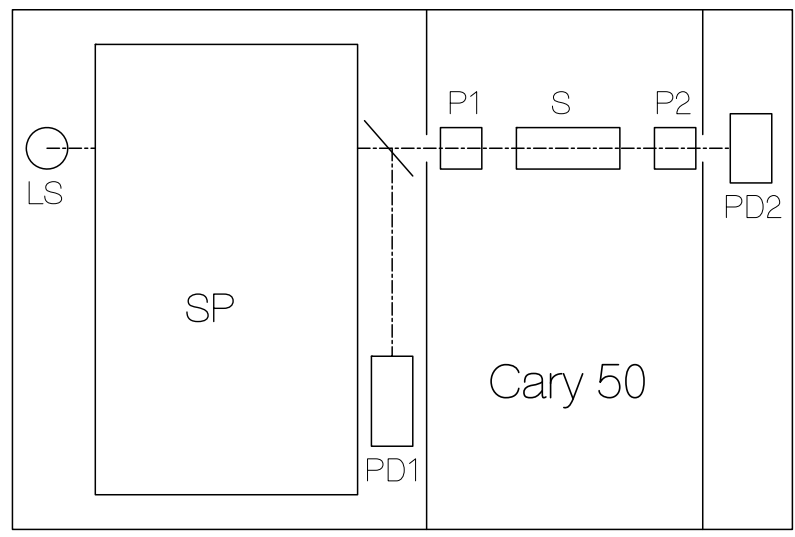

(b)

Figure 1. (a) Top part: Experimental setup for singlewavelength optical polarization rotation measurement of an optical active sample. LS: light source (here: He-Ne laser $\lambda$ $=632.8 \mathrm{~nm}$ ). P1: Glan polarizer aligned to horizontal polarized light transmission. P2: Glan polarizer operated vertical oriented (crossed to P1) and horizontal oriented (parallel to P1). S: optical active sample. PD: Photo-detector. DMM: Digital multimeter. Bottom part: Polarizer orientations of $P 1$ and $P 2$, polarization plane rotation for d-rotatory and l-rotatory samples, and light polarization direction after P2 (analyzer) are indicated. (b) Experimental setup for optical rotatory dispersion measurement of an optical active sample. In Cary $50 \mathrm{UV}-\mathrm{V}$ is spectrophotometer sample compartment, two Glan-polarizers, P1 and P2, aligned to crossed and parallel orientation, are inserted before and behind the position of sample S. Components of Carry 50 are: LS full-spectrum Xe pulse lamp; SP Cerny-Turner monochromator; PD1 and PD2 silicon diode detectors. where $S_{\text {out }}$ is the transmitted light power, $S_{\text {in }}$ is the incident light power, $T_{\mathrm{P} 1}$ is the transmission through polarizer $\mathrm{P} 1, T_{\mathrm{S}}$ is the transmission through the optical active sample $\mathrm{S}$, and $T_{\mathrm{P} 2}$ is the transmission through analyzer $\mathrm{P} 2$. The light transmission through the crossed polarizer arrangement with polarization rotation $\phi$ of the optical active sample is

$$
T_{\perp}(\phi)=T\left(\angle(\mathrm{P} 2, \mathrm{P} 1)=90^{\circ}, \phi\right)=T_{\mathrm{P} 1} T_{\mathrm{S}} T_{\mathrm{P} 2,0} \sin ^{2}(\phi) .
$$

The light transmission through the parallel polarizer arrangement is

$$
T_{\|}(\phi)=T\left(\angle(\mathrm{P} 2, \mathrm{P} 1)=0^{\circ}, \phi\right)=T_{\mathrm{P} 1} T_{\mathrm{S}} T_{\mathrm{P} 2,0} \cos ^{2}(\phi),
$$

where $T_{\mathrm{P} 2,0}$ is the transmission through $\mathrm{P} 2$ for parallel orientation of $\mathrm{P} 1$ and $\mathrm{P} 2$ in the case of $\phi=0$.

The transmission ratio, $T_{\perp} / T_{\|}$, is

$$
\frac{T_{\perp}(\phi)}{T_{\|}(\phi)}=\frac{\sin ^{2}(\phi)}{\cos ^{2}(\phi)}=\tan ^{2}(\phi) .
$$

Solving Equation (2) for the rotation angle $\phi$ of optical polarization gives

$$
\phi=\arctan \left[ \pm\left(\frac{T_{\perp}}{T_{\|}}\right)^{1 / 2}\right] .
$$

The positive sign applies to dextro-rotatory (= d-rotatory $=$ right-rotatory) samples. The negative sign applies to laevo-rotatory (= 1-rotatory = left-rotatory) samples. The rotation sign is determined by rotating the analyzer P2 slightly out of the crossed orientation by an angle $\delta$ $(|\phi|>\delta>0)$ and measuring the transmission ratio (see illustration in lower part of Figure 1(a)). For d-rotatory behavior one gets

$$
T_{\text {d-rot }}\left(\angle(\mathrm{P} 2, \mathrm{P} 1)=90^{\circ}+\delta, \phi\right)<T_{\text {d-rot }}\left(\angle(\mathrm{P} 2, \mathrm{P} 1)=90^{\circ}, \phi\right) \text {, }
$$

and for 1-rotatory behavior one gets

$$
T_{\text {1-rot }}\left(\angle(\mathrm{P} 2, \mathrm{P} 1)=90^{\circ}+\delta, \phi\right)>T_{\text {d-rot }}\left(\angle(\mathrm{P} 2, \mathrm{P} 1)=90^{\circ}, \phi\right) \text {. }
$$

The analysis above is unambiguous for optical rotation $|\phi|<90^{\circ}$ which is accomplishable by reduction of sample length and/or concentration of optical active molecules.

The experimental arrangement for the determination of the optical rotation of polarization as a function of wavelength (optical rotatory dispersion) is shown in Figure 1(b). The P1-S-P2 polarizer-sample-analyzer assembly is mounted in the sample chamber of the applied UV-Vis spectrophotometer Cary 50 from Varian. The wavelength range of this spectrophotometer is $190 \mathrm{~nm}$ to $1100 \mathrm{~nm}$.

In our experiments calcite Glan polarizers were used for P1 and P2. The polarizer P1 is oriented for horizontal 
polarized light transmission (after Cary 50 spectrometer horizontal polarized light component is more intense than vertical polarized light component). The light transmissions in the Cary 50 spectrophotometer through the horizontal oriented polarizer P1 alone (dashed curve), through the horizontal oriented polarizers P1 and P2 (solid curve), and through the horizontal oriented polarizer P1 and vertical oriented polarizer P2 (dotted curve) are shown in Figure 2. Below $390 \mathrm{~nm}$ the transmission through calcite begins to decrease remarkably and below $218 \mathrm{~nm}$ the transmission through calcite becomes diminishingly small (transmission behavior of calcite is found in $[7,8])$. For optical rotatory dispersion measurement in the wavelength range from $200 \mathrm{~nm}$ to $390 \mathrm{~nm}$ the calcite Glan polarizers should be replaced by $\alpha$-BBO Glan polarizers with better transmission performance $(\mathrm{BBO}=$ barium borate $\left.=\mathrm{BaB}_{2} \mathrm{O}_{4}\right)[9]$.

The Cary 50 spectrophotometer with polarimeter accessory of Figure 1(b) was applied to determine the molar optical rotation [1-5] of grape sugar (D-glucose) in Millipore water in the wavelength range from $220 \mathrm{~nm}$ to $820 \mathrm{~nm}$. Grape sugar from Frießinger Mühle, Bad Wimpfen (www.friessinger-muehle.de) was purchased in a supermarket and used without further purification. Its composition is D-glucose with crystal water, the sum formula is $\mathrm{C}_{6} \mathrm{H}_{12} \mathrm{O}_{6} \cdot \mathrm{H}_{2} \mathrm{O}$ (molar mass $M_{m}=198.18$ $\left.\mathrm{g} \cdot \mathrm{mol}^{-1}\right)$. It was dissolved in Millipore water. A mass concentration of $C_{m}=0.3 \mathrm{~g} \cdot \mathrm{cm}^{-3}$ was prepared (near saturation concentration) and used in the experiments. The

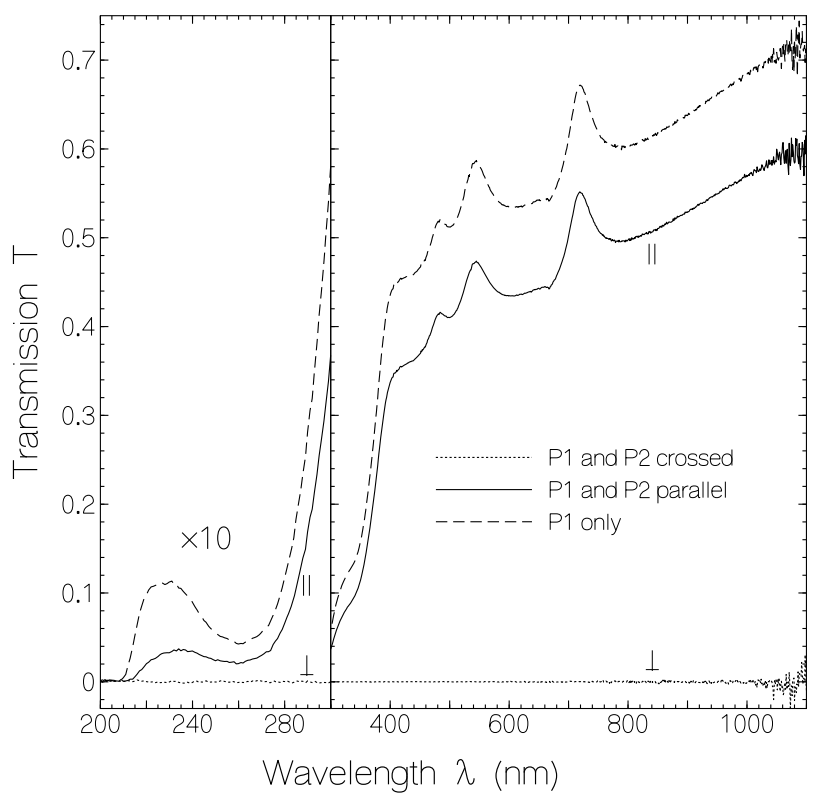

Figure 2. Transmission behavior of applied calcite Glanpolarizers in Carry $50 \mathrm{UV}-\mathrm{Vis}$ spectrophotometer. Transmission plane of polarizer P1 is oriented horizontal. For left part curves are expanded a factor of 10 . corresponding grape sugar molar density was $C_{M o l}=C_{m} / M_{m}=1.51 \mathrm{~mol} \cdot \mathrm{dm}^{-3}$ and the corresponding molecule number density was

$N_{m}=C_{M o l} N_{A}=9.12 \times 10^{20} \mathrm{~cm}^{-3} \quad\left(N_{A}\right.$ is the Avogadro constant). The experiments were carried out at room temperature $\left(\vartheta=21.5 \pm 0.5^{\circ} \mathrm{C}\right)$. The solution was measured in fused silica cells. In the wavelength range from 300 $\mathrm{nm}$ to $820 \mathrm{~nm}$ a cell length of $\ell=5 \mathrm{~cm}$ was used. In the wavelength range from $220 \mathrm{~nm}$ to $300 \mathrm{~nm}$ the measurements were carried with a cell length of $\ell=1 \mathrm{~cm}$.

Light transmissions, $T(\lambda)$, through the applied grape sugar solution in cell lengths of $\ell=1 \mathrm{~cm}$ (solid curve) and $\ell=5 \mathrm{~cm}$ (dashed curve) in the wavelength range from $200 \mathrm{~nm}$ to $820 \mathrm{~nm}$ are displayed in Figure 3. The inset shows the absorption cross-section spectrum $\sigma(\lambda)$ of the applied grape sugar in the UV spectral region. It was calculated by the relation $\sigma=-\ln (T) /\left(N_{m} \ell\right)$. Absorption shoulders at $325 \mathrm{~nm}$ and $215 \mathrm{~nm}$, and an absorption peak at $268 \mathrm{~nm}$ with $\sigma(268 \mathrm{~nm})=5.64 \times 10^{-22} \mathrm{~cm}^{2}$ are observed.

The angle (in radian) of which the plane of polarization rotates in an optical active sample of length $\ell$, vacuum wavelength $\lambda$, and refractive indices $n_{r}$ and $n_{1}$ is given by [1-5]

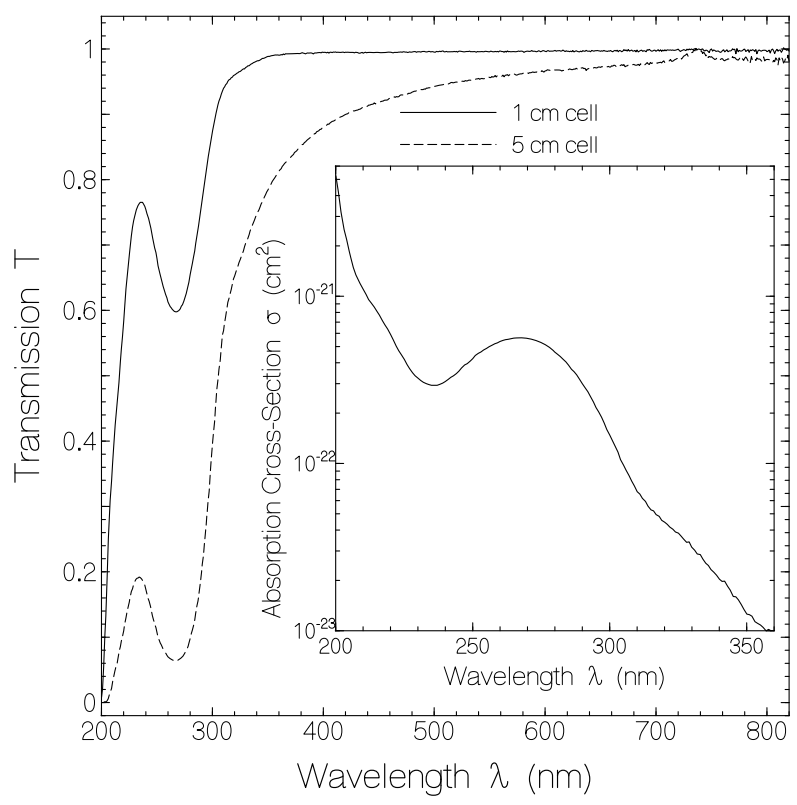

Figure 3. Transmission $T(\lambda)$ of grape sugar (D-glucose) in Millipore water. Mass concentration: $C_{\mathrm{m}}=0.3 \mathrm{~g} \cdot \mathrm{cm}^{-3}$ of $\mathrm{C}_{6} \mathrm{H}_{12} \mathrm{O}_{6} \cdot \mathrm{H}_{2} \mathrm{O}$. Molar mass of $\mathrm{C}_{6} \mathrm{H}_{12} \mathrm{O}_{6} \cdot \mathrm{H}_{2} \mathrm{O}: M_{\mathrm{m}}=198.18 \mathrm{~g}$ $\mathrm{mol}^{-1}$. Sample lengths $\ell=1 \mathrm{~cm}$ (solid curve) and $\ell=5 \mathrm{~cm}$ (dashed curve). Inset shows absorption cross-section spectrum $\sigma(\lambda)$ of grape sugar in Millipore water $\left(\sigma=-\ln (T) M_{m} /\left(\ell C_{m} N_{A}\right)\right.$ where $N_{\mathrm{A}}$ is the Avogadro constant). 


$$
\phi=\frac{\phi_{r}-\phi_{l}}{2}=-\frac{k_{r}-k_{l}}{2} \ell=\frac{n_{l}-n_{r}}{2} k_{0} \ell=\frac{\pi \ell}{\lambda}\left(n_{l}-n_{r}\right),
$$

where $k_{i}=n_{i} k_{0}=n_{i} 2 \pi / \lambda$ is the wavevector of right $(i$ $=r)$ or left $(i=l)$ circular polarized light $\left(k_{0}=2 \pi / \lambda\right)$.

The specific rotation $[\phi]$ of an optical active species in a sample is expressed in degrees $\left(1 \mathrm{rad}=180^{\circ} / \pi=180 / \pi\right.$ deg) and normalized to mass density or concentration $\rho$ of the optical active species in $\mathrm{g} \cdot \mathrm{cm}^{-3}$ and sample length $\ell$ in $\mathrm{dm}$. The corresponding numerical value equation is

$$
[\phi]=\frac{180}{\pi} \frac{\phi}{\rho \ell} .
$$

([ $\phi]$ is polarization rotation in deg for sample of density or concentration of $\rho=1 \mathrm{~g} \cdot \mathrm{cm}^{-3}$ and sample length of $\ell=$ $1 \mathrm{dm} ; \phi$ is in $\mathrm{rad})$.

The molar optical rotation $[m]$ of optical active molecules in a sample is expressed in degrees and normalized to the molar density or concentration

$\rho_{\text {Mol }}=1000 \rho / M_{M o l}$ in mol dm ${ }^{-3}$ and sample length $\ell$ in $\mathrm{m}\left(\rho\right.$ in $\mathrm{g} \cdot \mathrm{cm}^{-3}$ and $M_{M o l}$ in $\left.\mathrm{g} \cdot \mathrm{mol}^{-1}\right)$. The corresponding numerical value equation is [3-5]

$$
[m]=\frac{180}{\pi} \frac{\phi}{\rho_{M o l} \ell}=\frac{180}{\pi} \frac{\phi M_{M o l}}{1000 \rho \ell}=[\phi] \frac{M_{M o l}}{100} .
$$

([m] is polarization rotation in deg for sample of density or concentration of $\rho_{\mathrm{Mol}}=1 \mathrm{~mol} \cdot \mathrm{dm}^{-3}$ and sample length of $\ell=1 \mathrm{~m}$ ). The specific optical rotation as well as the molar optical rotation depends on the wavelength $\lambda$, the temperature $\vartheta$, the solvent, and in the case of sample dissociation or aggregation on the used concentration. The complete specification is therefore expressed in $[\phi]_{\lambda}^{\vartheta}$ (solvent, concentration in g per $100 \mathrm{~cm}^{3}$ ) or $[m]_{\lambda}^{\vartheta}$ (solvent, concentration in mol per $1 \mathrm{dm}^{3}$ ).

The wavelength dependence of the optical rotation in the transparency region is related to the absorption spectrum of the optical active sample by the Drude expression [1-5]

$$
\phi(\lambda)=\sum_{j} \frac{A_{j}}{\lambda^{2}-\lambda_{j}^{2}},
$$

where $\lambda_{j}$ is the wavelength of transition from the ground state to the excited state $j$, and $A_{j}$ is a constant depending on the absorption strength of this transition.

In the absorption region the absorption band linewidths $\Delta \lambda_{j}$ (FWHM) have to be considered and the Drude expression changes to the Cotton effect equation $[2,4]$

$$
\phi(\lambda)=\sum_{j} \frac{A_{j}}{\lambda^{2}-\lambda_{j}^{2}+\frac{\lambda_{j}^{4}}{\lambda^{2}\left(\lambda^{2}-\lambda_{j}^{2}\right)}\left(\frac{\Delta \lambda_{j}}{2}\right)^{2}} .
$$

(Equation 2.407 in [2] rewritten to $\lambda$ dependence using $\mathrm{d} v / \mathrm{d} \lambda=\mathrm{d}(c / \lambda) / \mathrm{d} \lambda=-c / \lambda^{2}$ leading to $\left.\gamma_{j}=-\left(c / \lambda^{2}\right)\left(\Delta \lambda_{j} / 2\right)\right)$.

\section{Results and Discussion}

The transmission measurement results through the polarizer-grape sugar solution - analyzer arrangement in the Cary 50 spectrophotometer are depicted in Figure 4. The concentration was $C_{m}=0.3 \mathrm{~g} \cdot \mathrm{cm}^{-3}$. The right part shows the transmissions through the cell of $5 \mathrm{~cm}$ length and the left part shows the transmission through the cell of $1 \mathrm{~cm}$ length. The solid curves belong to $T_{\perp}(\lambda)$ and the dashed curves belong to $T_{\|}(\lambda)$. The dotted curves shows the ratio $T_{\perp}(\lambda) / T_{\|}(\lambda)$. The shape of $T_{\|}(\lambda)$ is mainly determined by the transmission behavior of the calcite polarizers. The finite transmission $T_{\perp}(\lambda)$ is caused by the rotation of the polarization direction by the optical active D-glucose solution. The transmission ratio $T_{\perp}(\lambda) / T_{\|}(\lambda)$ is relevant for the calculation of optical rotation of polarization (Equations (2) and (3)).

The determined optical rotatory dispersion $\phi \times 180 / \pi$ (in degree) of the investigated grape sugar solution is depicted in Figure 5. It was obtained from Figure 4 by application of Equation (3). The left part belongs to a cell length of $1 \mathrm{~cm}$ and the right part belongs to a cell length of $5 \mathrm{~cm}$. The dash-dotted curve on the right part is the single-term regression fit of the Drude expression Equation (8) $\left(\phi(\lambda)=A_{0} /\left(\lambda^{2}-\lambda_{0}^{2}\right)\right)$ to the experimental

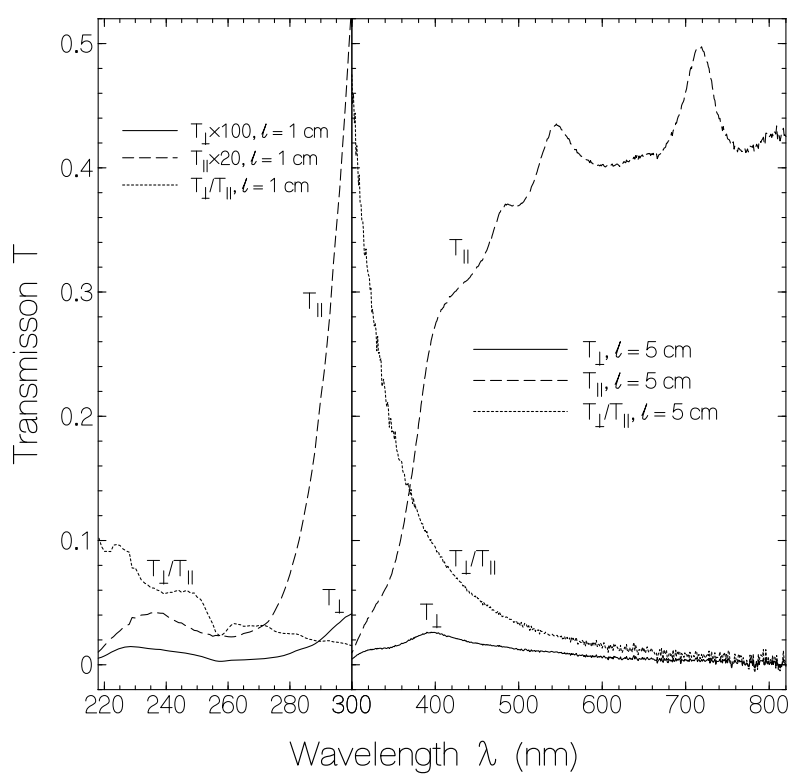

Figure 4. Transmission behavior of polarizer arrangement with inserted grape sugar sample. Mass concentration: $C_{\mathrm{m}}=$ $0.3 \mathrm{~g} \cdot \mathrm{cm}^{-3}$ of $\mathrm{C}_{6} \mathrm{H}_{12} \mathrm{O}_{6} \cdot \mathrm{H}_{2} \mathrm{O}$. Temperature $\vartheta=21.5^{\circ} \mathrm{C} \pm$ $0.5^{\circ} \mathrm{C}$. Left part: Sample length $\ell=1 \mathrm{~cm} ; T_{\perp}$ (solid curve) is expanded a factor of $100 ; T_{\|}$(dashed curve) is expanded a factor of 20. Right part: sample length $\ell=5 \mathrm{~cm}$. 


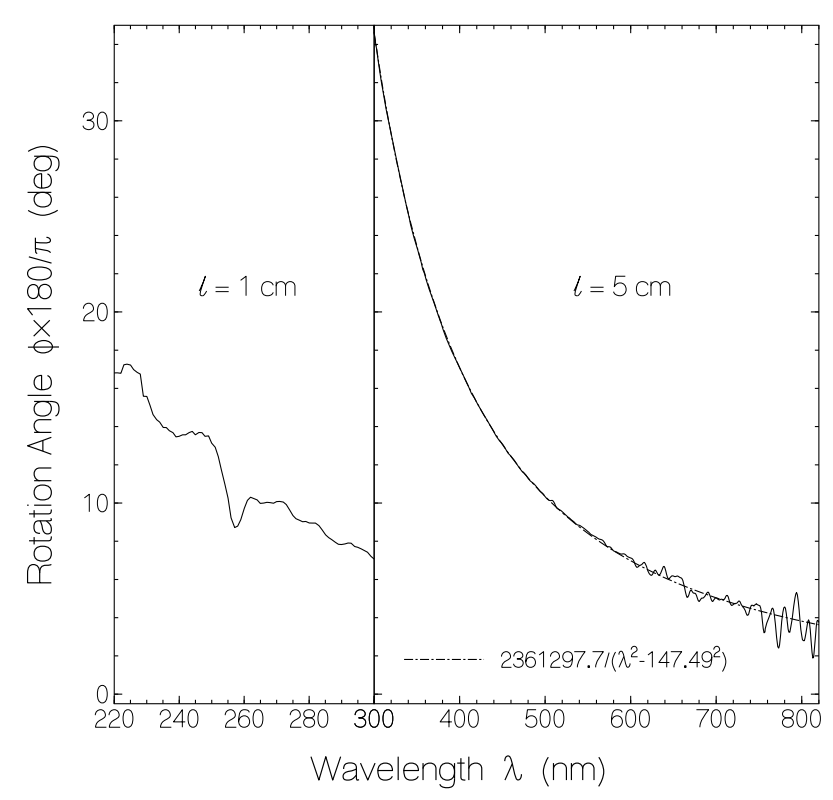

Figure 5. Optical polarization rotation $\phi$ of grape sugar samples in Millipore water. Mass concentration: $C_{\mathrm{m}}=\mathbf{0 . 3}$ $\mathrm{g} \cdot \mathrm{cm}^{-3}$ of $\mathrm{C}_{6} \mathrm{H}_{12} \mathrm{O}_{6} \cdot \mathrm{H}_{2} \mathrm{O}$. Left part: Sample length $\ell=1 \mathrm{~cm}$.

Right part: Sample length $\ell=5 \mathrm{~cm}$. Curves calculated from $T_{\perp} / T_{\|}$of Figure 4 (Equation (3)).

data. In the right region $(\lambda \geq 300 \mathrm{~nm}) \mathrm{D}$-glucose is transparent and the Drude relation fits well to the experimental curve. The apparent single oscillator transition wavelength is $\lambda_{0}=147.49 \mathrm{~nm}$ indicating a dominant apparent absorption band there. In the left region $(\lambda<300 \mathrm{~nm})$ the transmission through the calcite polarizers is small reducing the accuracy of rotation angle dispersion determination. There D-glucose is slightly absorbing (see Figure 3) causing a slight structuring of the optical rotatory dispersion curve due to the Cotton effect dispersion [1-5].

The obtained molecule specific molar optical rotatory dispersion $[m(\lambda)]$ (Equation (7)) of D-glucose is displayed in Figure 6. It shows the optical rotation in degree $\left(^{\circ}\right)$ of $1 \mathrm{M}\left(1 \mathrm{~mol} \cdot \mathrm{dm}^{-3}\right)$ glucose in a cell of $1 \mathrm{~m}$ length. Over the whole displayed wavelength range from $220 \mathrm{~nm}$ to $820 \mathrm{~nm}$ the dispersion fits well to the singleterm Drude formula. Some Cotton effect dispersion is seen in the D-glucose absorption region. The D-glucose absorption in the 220 to $300 \mathrm{~nm}$ region (see inset of Figure 3) is too weak to influence markedly the polarization rotatory dispersion which is determined by strong absorption in the vacuum UV spectral range.

The obtained optical rotatory dispersion of D-glucose in Millipore water is in good agreement with published results [10]. The here determined molar optical rotation at $\lambda_{D}=589.3 \mathrm{~nm}$ is $[m]_{D}^{21.5^{\circ} \mathrm{C}}$ (Millipore water, 1.514 $\left.\mathrm{mol} \cdot \mathrm{dm}^{-3}\right)=95.8 \pm 1 \mathrm{deg} \mathrm{mol}^{-1} \cdot \mathrm{dm}^{3} \cdot \mathrm{m}^{-1}$. The correspond-

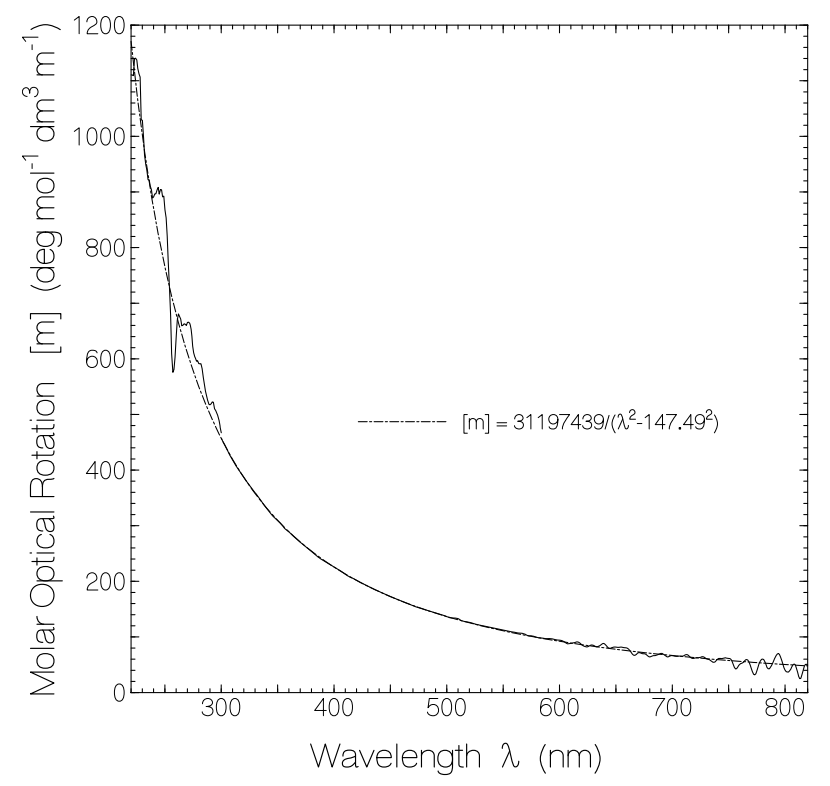

Figure 6. Molar optical rotation [m] of grape sugar in Millipore water. Curve calculated from curves of Figure 5 by use of Equations (6) and (7).

ing specific rotation is $[\phi]_{D}^{21.5^{\circ} \mathrm{C}}$ (Millipore water, 27.27 $\left.\mathrm{g} / 100 \mathrm{~cm}^{3}\right)=53.2 \pm 0.6^{\circ}\left(M_{m}=180.16 \mathrm{~g} \cdot \mathrm{mol}^{-1}\right.$ for D-glucose) is in good agreement with results found in the literature $\left([\phi]_{D}^{20^{\circ} \mathrm{C}} \quad\right.$ (water, $\left.\left.10 \mathrm{~g} / 100 \mathrm{~cm}^{3}\right)=52.7^{\circ}[11]\right)$.

\section{Conclusion}

A simple fixed-polarizer-analyzer polarimeter and spectropolarimeter were designed and applied to the measurement of the optical rotatory dispersion of D-glucose. In the arrangements the polarizer and analyzer are aligned perpendicular $\left(T_{\perp}\right)$ and parallel $\left(T_{\|}\right)$, and the rotation of the polarization plane is calculated from the transmission ratio $T_{\perp} / T_{\|}$. Besides two polarizers, no extra polarimeter and spectropolarimeter equipment are required for the determination of the specific optical rotation at a fixed wavelength or for the determination of the optical rotatory dispersion over a wide wavelength range.

\section{Acknowledgements}

The author thanks Prof. F. J. Gießibl for his kind hospitality.

\section{REFERENCES}

[1] L. D. Barron, "Molecular Light Scattering and Optical Activity," 2nd Edition, Cambridge University Press, Cambridge, 2009.

[2] E. Charney, "The Molecular Basis of Optical Activity. Optical Rotatory Dispersion and Circular Dichroism," John Wiley \& Sons, New York, 1979. 
[3] P. Grabbé, "Optical Rotatory Dispersion and Circular Dichroism in Organic Chemistry," Holden-Day, San Francisco, 1965.

[4] A. Moscowitz, "Theoretical Aspects of Optical Activity. Part One: Small Molecules," In: I. Prigogine, Ed., Advances in Chemical Physics, Vol. IV, Interscience Publishers, New York, 1972, pp. 67-112.

[5] S. F. Mason, "Molecular Optical Activity and the Chiral Discriminations," Cambridge University Press, Cambridge, 1982.

[6] E. M. Slayter, "Optical Methods in Biology," Wiley-Interscience, New York, 1970, Chapter 27.

[7] D. E. McCarthy, "Transmittance of Optical Materials from $0.17 \mu$ to $3.0 \mu$," Applied Optics, Vol. 6, No. 8, 1967, pp. 1896-1898.

[8] F. Wu, G. Li, J. Huang and D. Yu, "Calcite/Barium Fluoride Ultraviolet Polarizing Prism," Applied Optics, Vol.
34, No. 19, 1995, pp. 3668-3670. http://dx.doi.org/10.1364/AO.34.003668

[9] V. P. Solntsev, E. G. Tsvetkov, V. A. Gets and V. D. Antsygin, "Growth of $\alpha-\mathrm{BaB}_{2} \mathrm{O}_{4}$ Single Crystals from Melts at Various Compositions: Comparison of Optical properties," Journal of Crystal Growth, Vol. 236, 2002, pp. 290296. http://dx.doi.org/10.1016/S0022-0248(01)02216-3

[10] I. Listowsky, G. Avigad and S. Englard, "Optical Rotatory Dispersion of Sugars. I. Relationship to Configuration and Conformation of Aldopyranoses," Journal of the American Chemical. Society, Vol. 87, No. 8, 1965, pp. 1765-1771. http://dx.doi.org/10.1021/ja01086a027

[11] S. Budavari, "The Merck Index. An Encyclopedia of Chemicals, Drugs, and Biologicals," 13th Edition, Entry\# 4353 Glucose, Merck \& Co., Inc., Rahway, 1989, p. 699. 